\title{
Day Re-construction: Understanding How College Students Manage Their Time Through Self-monitoring
}

\author{
Haining Zhu, Jiawei Chen, Hongjian Wang, Sarah Almoaiqel, \\ Frank Materia, Xiying Wang, Natalie Cope, John Carroll \\ Pennsylvania State University \\ University Park, PA, 16801, USA \\ hbz5037@psu.edu
}

\begin{abstract}
With a plethora of possibilities for new social experiences, activities, and other prospects, college students may find it challenging to balance their time. To facilitate their awareness of and reflection upon time expenditures regarding the three pillars of a balanced life: work, personal maintenance and leisure. We designed and evaluated a web app called LifeLogger. This application harnesses semi-automated, self-tracking, and visualization features to support awareness and reflection of time use. We invited 13 participants to interact with the prototype for a week, and followed up with semi-structured interviews to understand their experiences of the application. We find that LifeLogger increases participants awareness and encourages self-reflection on time use, which could facilitate participants in comprehending their time expenditures. We conclude by discussing potential design strategies for time management.
\end{abstract}

\section{Introduction}

The "college years" can be one of the most exciting times in a young adult's life. For most traditional students (i.e., ages 18-24), the college years present a unique opportunity for individuals to experience independence for the first time. One of the unique challenges that comes along with this newly acquired independence involves students deciding how to spend their time. Learning to manage time for optimum individual benefit, or a "balanced lifestyle," is the key. Previous literature suggests that it is important to support college students in fostering balanced time management skills to improve academic performances [1], facilitate health and well-being [2], and develop meaningful use of time $[3,4]$. One major element to achieving a balanced use of time lies in the awareness of how time is spent. How- ever, college students often lack awareness of how they allot their time [2]. Facilitating students' awareness and reflection regarding personal time expenditure may thus enable them to better balance their college lives.

College students' daily activities and time can be categorized into three different "pillars" of a balanced life: work, personal maintenance, and leisure; these pillars have been studied as a framework for a balanced lifestyle [5]. Previous research has shown that students are not aware of how much time they spend studying and working, the first pillar [2]. College students' wellbeing may be hindered by assigning a low priority to the second pillar of personal maintenance, which entails all health aspects (e.g., diet, exercise, sleep, and hygiene) $[6,7]$. Similarly, imbalances regarding the third pillar hinder students' development and engagement in leisure activities, which are beneficial to students' academic performances and well-being [8].

A recent study focused primarily on leisure attempted to address lifestyle balance problems by encouraging awareness and reflection through time use tracking and visualization strategies across the three pillars of a balanced lifestyle [2]; they found that the tracking and visualization were highly burdensome, partly because they relied on manual paper-pencil tracking and data input into excel sheets, and because instantaneous feedback tools were not implemented. Our research goal is to design, deploy, and evaluate a system that harnesses commercially available sensor-based technology and leverages a semi-automated tracking approach [9]; we aim to help students better manage their time by facilitating their awareness and reflection on daily time spent.

This research uses design science methodology to contribute: 1) empirical understandings of college students' time expenditures framed using the three pillars 
of a balanced life, as well as how self-monitoring technology aided their awareness and reflection; 2) a smallscale evaluation of the LifeLogger system which demonstrates potential efficacy in promoting awareness and reflection. Our study builds upon existing literature by providing insights for designing technology which better accommodates college students' awareness and reflection toward time management. This presents opportunities for further research into college students' time expenditures and life balancing.

\section{Related Work}

Momentary Data Collection Methods Researchers have attempted to understand how college students distribute their time between study and leisure activities $[10,11,2]$. Many methods (e.g. survey, interview, diary) can be employed to measure how individuals construct their routines, but such methods require individuals to recall a series of points in their daily life, and may suffer from high burden and low level of recall accuracy. For example, Chen et al. required students to track all daily activities sequentially, and enter data manually into Excel [2]. Researchers have long discussed the inherent problem of recall bias for retrospective research [12].

Momentary data collection methods can improve data accuracy and offer finer-grained details. It often utilizes diaries (i.e, paper or electronic) in which users record daily data at preset scheduled times; these methods have allowed for insights into momentary variables, including individuals' feelings in their typical environments, with whom they spend time, moods and stress at particular moments, etc. These methods thus improve ecological validity of individual's collected data [13].

Day Reconstruction Method (DRM) is a technique widely adopted to assess experiences and affect in everyday life [14]. DRM involves the retrospective recall of the study period as a continuous sequence of episodes, and each episode is described by a series of attributes such as onset and duration, location, social interaction, and activity. Data collected by DRM have enormous utility, as they provide good measures of students' everyday behaviors and lifestyles. However, participants often find studies which utilize fine-grained incremental data collection to be burdensome [15, 13]. For example, participants are sometimes expected to track activities, durations, locations, accompaniments, emotions and stress in 30-minute increments [2]. As a result, many of these conducted studies have utilized fewer than 20 participants [13]. Self-tracking techniques, such as automated and semi-automated tracking [9], could provide opportunities to better enable the experience sam- pling method by lowering participants' tracking burdens. However, automated tracking has several unique drawbacks; it often provides trackers with decreased self-awareness, fewer opportunities for reflection [16], and less engagement [17]. In this study, we adopted DRM, design and developed an alternative to real-time, momentary sampling systems, and assess how college students distribute their time by utilizing the popular model of the 3 pillars of college.

Self-Tracking Technologies Self-tracking or selfmonitoring [18] is the the process of a person recording his or her own behaviors, thoughts, and feelings. While self-tracking has been an important clinical practice for assessment, diagnosis, and treatment [19], it has more recently become more available with the ubiquity of mobile devices. Many self-tracking applications with automated sensing features have been designed and developed, making it easier for people to collect and store personal data passively. Self-tracking applications can help people to reflect on their own data by analyzing patterns, presenting visualizations, and providing feedback at opportune times [20]. In their effort to increase awareness of individual behaviors and promote health behavior change, researchers and companies have designed self-tracking technologies for physical activity [21, 22, 23], sleep [24], and diet [25, 26].

In recognizing the pros and cons of both automated (i.e., pros include low burden and increased data accuracy [9]; cons include decreased self-awareness, selfreflection [16], less engagement [17]) and manual tracking (i.e., pros include evoking reflection, increasing awareness and accountability [16]; cons include burden [15] and low data accuracy [24]) approaches, Choe et al. [9] have suggested the semi-automated tracking method to combine the benefits of both approaches. For example, SleepTight [24] lower the burden of manually logging sleep related data by leveraging smartphones and wearable devices. Our work differs from prior works by focusing on the student-centered perspective and by using semi-automated self-monitoring technology to assess how college students distribute their time; we also identify opportunities for iterative improvements to further support student life balancing.

\section{LifeLogger}

We designed a web application called LifeLogger to promote student awareness and reflection on their college life. In designing the system, we focused on answering the following research questions:

1. What are students' intended time expenditures and their actual time expenditures? 


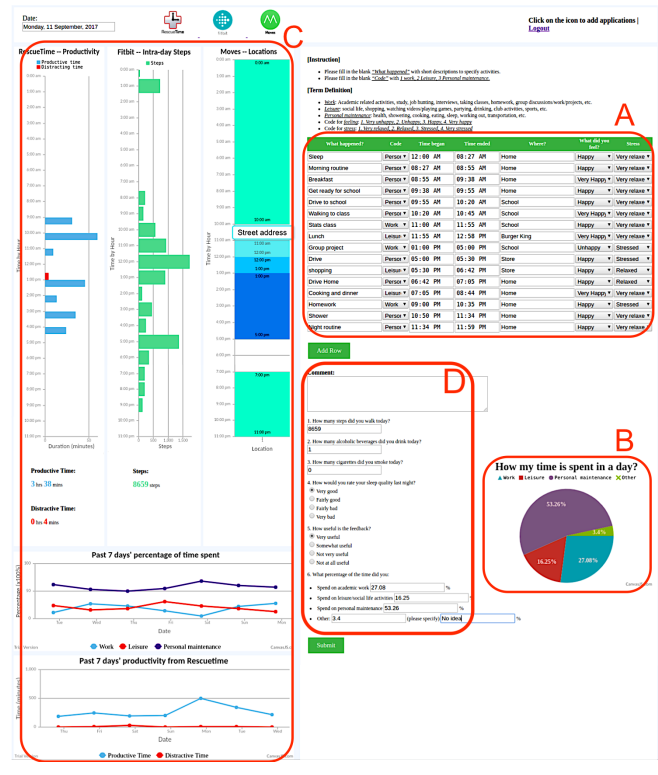

Figure 1: LifeLogger user interface. Participants were asked to import data streams from RescueTime, Fitbit and Moves, which would show detailed breakdowns of their their automatically collected data $(C)$. Part $C$ acts as an activity recorder prompt which helps participants answer a daily survey (A \& D). Results from Part A will appear in the form of a pie chart (Part B). At the bottom of Part C, there are 2 aggregated 7 day data summaries; the first is generated from Part B. Below that, we displayed the participant's weekly RescueTime usage in the form of a line graph. *Note: Data shown in this example is fictitious and does not represent data from our participant.

2. How can self-monitoring influence students' awareness of their time expenditures?

3. How does data visualization aid students in retrospectively reflecting upon their time expenditures?

\subsection{Design Strategies}

3.1.1 Designing for Increased Awareness: LifeLogger's first intended goal is to increase students' awareness of their time expenditures across the three pillars of college life (i.e., work/academics, personal maintenance, and leisure). To do so, we provided clear definitions which categorized students' daily activities, and asked participants to categorize their daily activities based on our definitions during their manual logging (Fig 1A). We define work as actions students undergo in the course of academic related pursuits (e.g., studying, jobs, interviews, classes, homework, group projects/work, etc.). Leisure encompasses the social life, shopping, watching movies/TV, playing games, attending parties, club activities, and group sports. Personal maintenance includes activities like hygiene, cooking, eating, sleeping, exercising, personal transportation, etc. Other indicates unlogged time spent switching between each activity (Fig 1). By increasing awareness, we aim to encourage college students to reflect more on their time management, and to aim to achieve more balanced, healthier lifestyles.

3.1.2 Designing for Self-reflection: Our design uses 3 layers to aid participants' daily activities reflection.

We asked students to import data streams from RescueTime, Fitbit and Moves. We chose these 3 applications because each provides some hint to understanding students' time expenditures. RescueTime, for example, shows how students have used their time, thus indicating whether they were studying (i.e., work/academics) or having fun (i.e., leisure). Fitbit tells students when they are active, and Moves records location changes throughout the day. To combat forgetfulness and recall bias, we provide automatic data visualizations from the 3 sources (Fig 1C) which students can use as prompts which aid in remembering events.

We intentionally designed LifeLogger to ask students to manually log their activities (Fig 1A). We intended that students would check the visualizations we provided. In addition, to help students better understand the automatically collected data, we aligned all visualizations into a 24 hour format. Our strategy to direct their thinking processes was to facilitate students to be able to check their computer usage, activities and locations in a linear, timely manner. Note that instead of a traditional map view (i.e., 2D and focused on location changes), we designed color-coded address blocks to align with times from Moves. We intended for this to facilitate students in considering how they spent their time across a day; we believed that students noting where they were located at specific times would elicit stronger and more reliable retrospective reflections of behaviors if the data were visualized meaningfully.

Finally, students need to understand their granular and continuous timeline of daily activities (Fig 1 B\&C) to answer the survey questions (Fig $1 \mathrm{~A} \& \mathrm{D}$ ).

We are aware that automatically collected data may not reflect actual events as they happen during a day. Therefore, we designed manual logs to allow students to have some autonomy to correct and reflect upon tracking data. In this way, we reinforced our 3 layers for reflection regarding their self-monitoring data. 


\subsection{LifeLogger Design}

The LifeLogger system consists of two components: a dashboard and a daily survey. Our design utilized semi-automated tracking [9] and daily surveys based on the Day Reconstruction Method [14].

Dashboard:Using APIs from RescueTime, Fitbit, and Moves, we incorporated automatically captured data and designed personalized feedback, which is displayed on the left hand side of the interface (Fig 1). RescueTime is a prominent commercially-available productivity tracking tool which automatically monitors students' computer usage. RescueTime utilizes five categories (very productive, productive, neutral, distracting, very distracting) to evaluate time use, which we revised to utilize only productive and distracted categories [17]. To do so, we combined very productive, productive, and half of the time on neutral into our productive category; the remainders were synthesized into our distracted category. Moves [27] is a location tracking tool which provides participants with their location data and daily physical activity feedback, and Fitbit [28] is a wearable device for tracking health-related data to provide participants with their physical activity metrics (e.g., steps).

Daily survey: We designed our daily survey based on DRM [14]. To better support participants' reflection, they were presented with data visualizations of the automatically collected data (Fig 1C), and asked to reflect upon the information provided to them to elicit deeper levels of reflection [29]. To achieve this, we aligned the feedback time with the survey table time, and then asked students to respond to a daily survey which asked them to enter their activity times, types, and durations, moods, and stresses on the right side of the interface (Fig 1 A\&D). A pie chart (Fig 1B) displays the results of the daily survey and indicates the distribution of a student's time in percentages.

\subsection{LifeLogger Implementation}

We implemented LifeLogger as a web-based service. The back-end is implemented with the Python Flask framework, which takes charge of: user authentication, access management, and data retrieval (Fig 2).

User authentication. Users are required to register with LifeLogger so we can securely provide visualizations of their data. The backend stores all user authentication information in a server-side SQLite database.

Access Management. Users authorize LifeLogger to retrieve their data from Moves, RescueTime, and Fitbit. All three external commercial services rely on OAuth authorization to securely share data with LifeLogger. In the backend, we will store the OAuth access

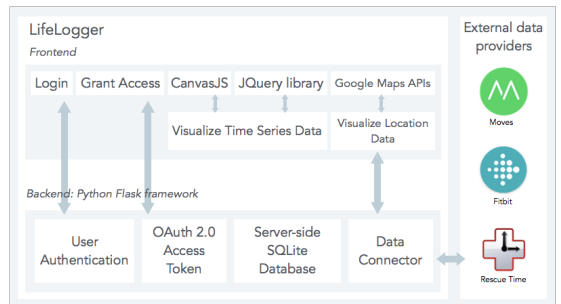

Figure 2: LifeLogger website architecture

tokens from different external services to our server-side SQLite database.

Data retrieval. Upon user request, the backend server will retrieve the user's data from corresponding services with the access token. The data are sent to the frontend for visualization in the user's browser.

Our frontend relies upon the JQuery library, CanvasJS, and Google Maps APIs for visualization. On the dashboard, various data from the three services asynchronously load through AJAX. Once data is available, Fitbit and RescueTime data are visualized with CanvasJS as a time series, and Moves data are visualized via Google Maps.

\section{Method}

To understand how semi-automated self-tracking technologies support college students to manage their time regarding the three pillars of a balanced life, and to explore how students reflect upon theimaler data through visualizations, we performed a field deployment study with college students at a Northeastern U.S. University. The study is approved by the Institutional Review Board (IRB). We sought volunteers from the university website for the following eligibility criteria were as follows: full-time undergraduate student studying at the aforementioned campus location; ages 18-24 years; proficiency with the English language; ownership of a smartphone with data capabilities; ownership of a laptop with internet access that is commonly used to browse the internet; willingness to install apps on personal devices for the study duration. We recruited 13 participants (10 females \& 3 males). Participants' ages ranged from 18 to 22 (mean $=20.77$ ). Every year of college is represented by at least one participant (Table 1).

\subsection{Study Procedure}

We organized our study procedure into three main components: a pre-study session, the deployment of LifeLogger, and a post-study interview.

Pre-study Session. All participants attended a prestudy session (15 min) before using LifeLogger, during which we introduced the study and procedures. Af- 
ter giving consent to participate, participants installed Moves and Fitbit apps on their smartphones, and RescueTime on their laptops. Participants were given a tutorial on how to use the system, and were instructed to not visit these apps or other commercial services for separate feedback when using LifeLogger.

Deployment. After the pre-study session, participants started to use LifeLogger for one week. We felt that a one week deployment would be sufficient to understand participants' first reactions using this tool and enable us to iteratively revise our design. During the deployment period, we sent emails to each participant at $10 \mathrm{pm}$ every night. Upon receiving the email notifications, participants logged into LifeLogger system. They filled out the tracking form assisted with automatically collected data, including location (from Moves), steps (from Fitbit), and computer usage (from RescueTime). After completing daily tracking forms in LifeLogger, participants were shown pie charts showing percentages of time spent in each category that day (work, leisure, and personal maintenance).

Post-study Interview. To evaluate our system, we held a semi-structured interview after completion of the one week study. To answer our research questions, interview questions included topics regarding how participants understood a "balanced lifestyle," what did they learn from using the system, how they viewed or managed their time before and after using LifeLogger, if they felt that participation changed any of their habits, and their opinions about the automatic data and visualization feedback. Participants are paid $\$ 15$ for the interview. After the experiment, the system is still available for them to access for a month.

Table 1: Participant demographics and majors.

\begin{tabular}{|c|c|c|c|c|}
\hline ID & Age & Sex & Year & Major \\
\hline P1 & 20 & F & 2 & Biobehavioral Health \\
\hline P2 & 22 & F & 4 & Economics \\
\hline P3 & 22 & F & 4 & Biology \\
\hline P4 & 18 & F & 1 & Business \\
\hline P5 & 21 & F & 4 & Psychology \\
\hline P6 & 21 & F & 3 & Recreation, Park\&Tourism Mgmt. \\
\hline P7 & 21 & F & 3 & Public Relations \\
\hline P8 & 21 & M & 4 & IT, Finance \\
\hline P9 & 19 & F & 2 & Math \\
\hline P10 & 22 & F & 4 & Finance \\
\hline P11 & 22 & F & 4 & Biobehavioral Health \\
\hline P12 & 22 & M & 4 & Energy, Business \& Finance \\
\hline P13 & 19 & M & 1 & IT \\
\hline
\end{tabular}

\subsection{Data Analysis}

We transcribed every audio recording. Each interview ranged from thirty to seventy minutes. For confidentiality purposes, we assigned a unique participant
ID (P\#) to denote participants. To understand changes in participants' awareness and reflection in practice, we first established properties of what they said without relying on existing theories (i.e., open coding); we then used thematic analysis [30] to identify, analyze, and report patterns within the qualitative data, generating and refining categorical codes using an inductive approach. This approach allowed us to understand how self-tracking influenced participants' awareness through data visualization, and how providing a granular and continuous timeline of daily activities helped participants to retrospectively reflect upon their behaviors. Four researchers separately and independently read and coded about $25 \%$ of the transcripts before then discussing the themes and reaching an agreement with the other researchers. After this step, one researcher coded and organized all of the interview data.

\section{Findings}

\subsection{RQ1: Estimated vs. Actual Expenditures}

Figure 3 shows a summary of what participants reported about their time management backgrounds. For work and leisure, participants' estimated time expenditures matched actual expenditures. However, personal maintenance showed a great difference between estimated and actual time expenditure. Participants attributed this to not having a clear idea about what constituted personal maintenance prior to the study. Although all participants were informed about definitions in the pre-session, only 2 participants considered sleep to be personal maintenance; this resulted in participants' selfreported time expenditures for personal maintenance differing significantly from their actual times. Despite the personal maintenance issue however, students in general noted that using the LifeLogger system helped them to more objectively understand their behaviors and made them more aware of their sometimes incorrect intuitions. "I thought I was doing this but I really wasn't or like I knew I was doing this too much and now I can prove that I was doing it more than I wanted to.” [P6]

\subsection{RQ2: Awareness and Life Balance}

\subsubsection{Improving Self-knowledge of Time Spent.} After tracking their daily activities for a week, participants reported that they gained a better understanding of how their time was spent. In the post-study interview, 4 participants reported that they were "completely wrong" about their time spent in the past. 9 of the other participants said that they were "a little bit off" about their time spent in the past. Several participants mentioned that by tracking their daily activities, they realized that 
they didn't spend as much time on work as they had prior thought. For example, P3 said: "I was just super shocked in the beginning. I thought that I spent more time on work." P11 described it in more detail, "there are some days where I work the whole day, and then there's other days like I think that I did a lot of homework, but then when I look at what I did all day, my work wasn't really a lot compared to everything else."

8 participants mentioned that they were surprised that they spent much more time on personal maintenance than they expected. For example, P3 explained further that personal maintenance activities are usually many small things that people tend to ignore easily: "I didn't really think about my time cooking, getting ready, showering, I didn't really think about that time." Participants believed that LifeLogger's feedback in part helped them to draw these conclusions and better see how they used their time. "I would say I was a little bit more aware of the things that I'm doing, and how to managing my time, because I was being able to see." [P1]

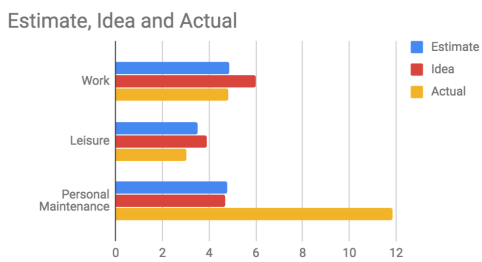

Figure 3: Comparison of participants' estimated, ideal, and actual hours spent per day performing actions in each of the three pillars.

5.2.2 Defining a Balance. Ultimately, students have their own definition of "life balance" based on unique values and goals. P10 offered a personal definition: "I define a balanced lifestyle for myself to be one that I give enough time towards personal maintaining myself personally and physically healthwise but without the extent of sacrificing time that I'd like to commit towards work or leisure. Basically getting the best of all three worlds would be the ideal lifestyle for me." P13 discussed how he valued the importance of leisure and social life, mainly as a support for work and emotional health: "[Leisure] makes me happy, and then it helps me at work because I'm happy when I go to work, and then it helps me sleep, because I know now I'm happy. I sleep better and work better." P6 also noted that the definition for a balanced lifestyle can vary based on plans and changing workloads: "[It's] finding a way to be able to do everything you want but to an extent. So like obviously there are days where you have to put work higher than leisure but maybe you do a little more leisure in another day where you don't have as much work."
5.2.3 Awareness of How Goals Affect Balance. We find that different participants perceived the importance of work, leisure, and personal maintenance with different motivations and purposes. When asked about their goals for enrolling in the study, 7 participants reported that their main goals included to improve academic performance, while 5 wanted to improve health/personal maintenance. Only 1 participant reported aiming for a balance of time between the three pillars. However, none of the participants mentioned improving leisure/social life as an important goal of participating in this study. Students explained that the perceived importance of each different aspect of college life was closely related to their goals. After the study, students demonstrated a greater awareness of how their values and goals affected time allocation. For example, work was the most important aspect for P9, and her goal was to prepare herself for the real world: "I feel like, now, there's a lot of benefit to me working very hard at this point in my life. Like college students in general, the more productive we are now, the more return our investment will have...I feel like there's a better return on our investment now of working hard, than there would be in a day to day job later on in life."

5.2.4 Enhancing Awareness and Evoking Reflection. While participants gained knowledge about how they spent their time by tracking daily activities, this also made them more interested in understanding their time spent, and encouraged them to reflect on it. For example, P4 mentioned: "I didn't realize how long I spend walking places all day on campus, I was never really interested [or] knew the break down, after I learned the break down, I became more interested in how I spend my time." Participants indicated that reflecting on time spent encouraged them to think about how to improve time management. P10 said: "I think it did affect how my time will be changed, [and] how my time will be managed in the future just because it did kind of planted a seed in me made me realize how my time was really broken down."

P9 described that self-tracking also encouraged her to manage her time in a more balanced way: "I feel like I will improve my time management. [I]t was just an eye opening type of experience. Because I realized the gaps that I have during my days." P6 gave an example that self-tracking helped her to manage her time better by encouraging her to focus more on work and study: "It helped me to time manage with my classes, so it made me focus more on 'ok I need to study more' or 'I really should do more my work I have projects coming up, so I really need to dedicate time to that.' ", 
Participants' self-tracking activities evoked not just reflection, but also deeper levels of reflection. Students compared the automatically collected data with the pie chart and noted feeling that the pie chart may be inaccurate in comparison. 7 participants expressed doubts about the accuracy of the final percentages as displayed in the pie chart. For example, P6 felt as though the process of capturing their data could cause errors. "[I]f I said I go to bed at 11 and I put it at 10, I might use the time to do something leisurely. So it might not be the most accurate if I said I did something then I switched the activity that I originally thought I was going to do."

\subsubsection{Promoting Intention for Behavioral Changes.}

LifeLogger appeared to increase participants awareness of behaviors; participants noted that the system helped them make correlations between behaviors and time management to achieve a desired outcome. P2 noted that, "I think I wouldn't want to add more work but when I was doing something on the computer I realized don't go on Facebook while trying to write a paper at the same time." Therefore, the system allowed participants to be aware of potentially undesirable habits and thus engage in more productive behaviors. In part, increased participants' awareness of actions promoted a sense of accountability for behaviors and balancing time. "I feel like I was held accountable for what I was doing...I think it definitely made me more aware." [P5] Awareness and accountability in conjunction allowed participants to employ self-negotiation to determine goals and schedules and potentially make corrections towards their desired time balances. "[I]f I spent five hours school work on a Monday and I got three things done for the week, then it took the pressure off of Tuesday, and I would say OK I'm doing this assignment Tuesday" [P5] However, participants might not want or be able to employ self-negotiation to reach goals unassisted. P9 would have preferred if LifeLogger offered recommendations to reach goals, suggesting that "[I]f you saw I was doing a lot of leisure maybe recommend hey go to the gym... give me advice on what would be productive." A participant was able to enact a behavioral change and reach her intended time allotment goals. "I changed my location because I knew if I was home I was more inclined like 'oh it's kind of getting late, I should probably go to bed.' But I was like, 'no, I need to study.' So I'm going to study somewhere else that could help me." [P9] This instance shows the intended use of our research; P9 used this technology to increase her selfawareness, to find correlations, to use those correlations to determine how to improve her behavior and time balance, and actually enact that plan for her desired out- come. Therefore, LifeLogger offered the possibility of increasing student intention to enact behavioral change.

\subsection{RQ 3: Reflection with Granular Data}

5.3.1 Recollection from Context. Participants have their own mental models to understand and analyze provided data. When doing so, participants found the contextualized data from Moves promoted meaningful reflection and recollection of not only locations, but behaviors and actions at a given time. "It does help me to remember like what exactly I was doing, to think about where I was at that time." [P12] 11 participants used Moves data in conjunction with other data (i.e., Fitbit and RescueTime) to reflect upon their actions. "My eyes just immediately go to where I was and then I remembered what I was doing then. And sometimes I would look at the Fitbit steps and be like 'oh I was walking a lot here', I must've been walking to class or something. But I would mostly use the Moves to see I'm at this point on campus now, or 'oh I went home for this amount of time' and it would help me because it would have a period of time that maybe I was in my apartment, and I'd be like 'OK what was I doing in my apartment?' " [P2] These automatic data work as cues for students to remember what they were doing at a given time frame.

5.3.2 Time Allocation Visualization. Students could more easily interpret outcomes when data presentations were simple and interactive. Most participants favored the pie chart (Fig 1B) to all other data visualizations provided, and all students reported that it and the time percentage breakdown were the most useful for interpreting their data. 10 Participants believed that the pie chart was one of the more effective ways to view time usage, and that it helped them reflect upon what had been done. P12 noted, "I like the pie chart the most ... it represented the whole day, and it's really easy to read. Like it gives you a good idea of how much of your day you spent doing something." The pie chart also helped participants to feel reportedly higher satisfaction and achievement on days in which they did more work. P6 stated that "[The pie chart] is kind of a nice way to reflect about your day, and it's satisfying almost because you're like 'wow I really did have a productive day' and it makes you feel good. It makes you go to bed feeling a little better about how you spent your day as opposed to just being like super frazzled...this helps [I] know what I'm just focusing on today. I don't have to think about yesterday or tomorrow or what I need to do next week. It's more like this is what I did, this is what I accomplished, now I can relax and go to bed and start over the next day."

When participants could see that they had achieved 
a balance on their data visualizations and were satisfied with how their time was spent, LifeLogger became a tool for reassurance, which may have bolstered motivation to continue using the tool. "For the days I get a pretty equal amount, like I get a pretty good amount of leisure, and also spent enough time on getting my work done too, I would usually feel satisfied." [P13] Participants who had felt that they had created an imbalance in their weekly time allocation (e.g., those who spent more time working and being productive) may even plan to correct the imbalance (i.e., by increasing leisure time at a later date). "[I]f I looked at that on a weekly basis I could see this "oh this day I spent more time doing this so maybe the next day I'll have more time for leisure'." [P2]

\subsubsection{Mixed Attitudes toward Levels of Data Detail.}

Participants had clear preferences for data from one specific application, or used limited features in conjunction. Notably, individuals' attitudes and preferences were not always consistent. Some prefered a minimalistic style; for example P5 stated, "I think there's a lot going on so it's hard to look at everything. I didn't really look at those [Automatic data]...this [Pie chart] was just right there in front of you, when you log everything, it was updated as you were typing stuff in."

Contrary to desiring minimalistic or simple data feedback, 5 participants expressed that simply understand how they spend their time seems to be insufficient. P8 noted that he would like to see more detailed feedback from the aggregated weekly line graph. "I feel this [line graph] is not particularly representative, I need more detailed data. For example, if I spent 16\% [of my day] in work, what specific work did I do? Did I spent those time on taking notes, or on finishing project? Only see the lines are not comprehensive enough for me."

Participants also think adding customizable, personalized goals would be more useful. P9 stated that, "On this [aggregated] line graph, I compared the actual line with what I imagined it should look like and calculated the deviation." She also suggested separating work, personal maintenance, and leisure from each other, because she only wanted to improve her time spent on work. She therefore felt that she did not need to have those categories together to meet her personal goals, and that she hardly paid attention to the extraneous data from the other categories.

\section{Discussion}

In the previous section, we highlighted how each component of LifeLogger facilitated students' awareness and retrospective reflection upon their time usage, and we noted a difference between students' intended and actual time expenditures. We will now discuss students' inaccurate time balance estimations in greater detail, how LifeLogger can promote student awareness and reflection, and potential design changes which would facilitate students in comprehending their time balances and reaching personal goals.

LifeLoggers most useful components for facilitating students' end of day reporting, and thus their time management skills, were the visualizations which displayed their time expenditures. Students displayed inaccurate estimations of time expenditures for each of the 3 categories. Their biggest errors occurred within the category of personal maintenance. Students generally felt shocked to see that they had spent as much time in this category as they had. We could attribute part of this phenomenon to participants not having a clear understanding about what constituted personal maintenance. However even the other categories, on which students had a better grasp, displayed a mismatch between estimated and actual expenditures. This suggests that a lack of comprehension is only a part of the problem, and that a lack of awareness or recall bias [12] may have prevented students from making more accurate estimations. Although this is an interesting avenue to follow, our research was not aimed at discovering the mechanism behind faulty time balancing estimations; future research will be needed to more thoroughly explore that issue.

Whatever the causes behind students' faulty estimations, estimations were faulty. Students may have believed they were primarily performing work activities at school, but through LifeLogger's prompting for detailed activity recording, as well as automatically collected location, computer usage, and steps data, they were better able to reflect and remember each activity change; this allowed them to see how even small periods of time spent doing non-work related activities at school could add up and become significant. Students' ability to see actual time expenditures showed them that they were performing more than just work related activities while at school. Once participants were made aware of their time imbalances, they could enact behavioral changes to improve time balance based on their personal goals. We saw this as a reality with 5 of our participants, but we believe that feedback from our participants could be used in future iterations to better facilitate this process. For example, many participants discussed wanting LifeLogger to be more customizable, especially with respect to data and goals. If we allowed participants to set goals for time allotment per day or week, we could perhaps utilize the scheduling of events (e.g., watching a new episode of a show on a certain day in the upcoming week 
for leisure, studying in a group session for a project for work, or sleeping for personal maintenance), to inform a recommendation for time management that would allow participants to reach their set goals. Such a process would enable those who cannot easily self-negotiate to benefit from the system.

We could also potentially better inform participants of their time expenditures by utilizing more subtle or nuanced methods of monitoring changes in activities, such as by using other physical activity sensors to more accurately and completely record participants' activities. However, we believe this approach might meet with heavy resistance as it could infringe upon privacy. Furthermore, we believe that awareness of time expenditure is more beneficial for students than providing complete and accurate reports of activities. Therefore, we recommend utilizing an approach which helps students become more aware of their actions as they take them, rather than a purely retrospective approach facilitated by technological recording.

Limitations. Our study had a small sample size and a short duration, and we purposefully sampled students that were self-motivated and interested in self-tracking. A short duration is not inherently a usability study limitation, but we must be cautious extrapolating longer behavioral trends from short uses. In addition, the majority of participants across the studies $(\mathrm{N}=13)$ were female $(\mathrm{N}=10)$. Results are therefore unlikely generalizable to the entire undergraduate population. Moreover, we did not quantitatively analyze each activity and feeling association because most students reported that they did not have strong feelings. Finally, although we did brief participants on the definition of personal maintenance activities, their lack of clarity on the issue may have affected the degree of error in their estimations, and thus the accuracy of that element of our work.

Future Work. Future studies could include a more diverse sampling with a higher number of male endusers, or investigate why students lack strong feeling associations, and thus how to better design means of measuring their emotions. More work regarding how selftracking burdens may affect adoption and system use may also be needed. However, as an exploratory experiment, we have gained valuable insight from our small deployment study. Further research should be aimed at finding out how to meaningfully visualize data in a specific context for intended goals, like reflection. Researchers could examine why the pie chart and color coded addresses succeeded while other similar visualizations, like the maps and line graphs, did not. Finally, our project had a short duration (i.e., 7 days), as stu- dents schedules are based on weeks. This resulted in students having actionable behavior change plans, but did not offer time to enact such plans. It further does not demonstrate how students would use the LifeLogger system over long periods of time. Longer study durations could examine how actionable plans are utilized by students, and if short term benefits noted in this work extend over longer durations.

\section{Conclusion}

Facilitating reflection and awareness regarding daily time expenditures in students has the potential to aid them in achieving healthier behaviors and more balanced lifestyles. The LifeLogger system utilizes a semiautomated self-monitoring approach to promote awareness and reflection in college students regarding their daily time expenditures. As the study shows, this tool harnesses visual cues (e.g., pie charts) from automatically tracked data (e.g., computer-usage, steps, location) to elicit individual reflection for manual data entry at the end of each evening. Results indicate that LifeLogger helps to promote reflection in college students, as all participants noted its utility in assisting them with becoming more self-aware of how effectively and efficiently they spend their time.

\section{References}

[1] S. Brint and A. M. Cantwell, "Undergraduate time use and academic outcomes: Results from the university of california undergraduate experience survey 2006," Teachers College Record, vol. 112, no. 9, pp. 24412470, 2010.

[2] H.-Y. Chen, C. Yarnal, J. T. Hustad, and D. Sims, "Take a selfie of life: A qualitative exploration of college students' self-reflections on free time use and personal values," Journal of College and Character, vol. 17, no. 2, pp. 101-115, 2016.

[3] R. Misra and M. McKean, "College students' academic stress and its relation to their anxiety, time management, and leisure satisfaction," American Journal of Health Studies, vol. 16, no. 1, p. 41, 2000.

[4] M. Trueman and J. Hartley, "A comparison between the time-management skills and academic performance of mature and traditional-entry university students," Higher education, vol. 32, no. 2, pp. 199-215, 1996.

[5] G. Kielhofner, "Temporal adaptation: A conceptual framework for occupational therapy.," American Journal of Occupational Therapy, 1977.

[6] K. Silliman, K. Rodas-Fortier, and M. Neyman, "A survey of dietary and exercise habits and perceived barriers to following a healthy lifestyle in a college population," Cal J Health Promot, vol. 18, p. 281, 2004. 
[7] M. T. Trockel, M. D. Barnes, and D. L. Egget, "Healthrelated variables and academic performance among first-year college students: Implications for sleep and other behaviors," Journal of American college health, vol. 49, no. 3, pp. 125-131, 2000.

[8] P. A. Bloland, "Leisure as a campus resource for fostering student development," Journal of Counseling \& Development, vol. 65, no. 6, pp. 291-294, 1987.

[9] E. K. Choe, S. Abdullah, M. Rabbi, E. Thomaz, D. A. Epstein, F. Cordeiro, M. Kay, G. D. Abowd, T. Choudhury, J. Fogarty, et al., "Semi-automated tracking: A balanced approach for self-monitoring applications," IEEE Pervasive Computing, vol. 16, no. 1, pp. 74-84, 2017.

[10] R. Arum and J. Roksa, Academically adrift: Limited learning on college campuses. University of Chicago Press, 2011.

[11] — Aspiring adults adrift: Tentative transitions of college graduates. University of Chicago Press, 2014.

[12] E. Hassan, "Recall bias can be a threat to retrospective and prospective research designs," The Internet Journal of Epidemiology, vol. 3, no. 2, pp. 339-412, 2006.

[13] A. A. Stone, J. E. Schwartz, D. Schkade, N. Schwarz, A. Krueger, and D. Kahneman, "A population approach to the study of emotion: Diurnal rhythms of a working day examined with the day reconstruction method.," Emotion, vol. 6, no. 1, p. 139, 2006.

[14] D. Kahneman, A. B. Krueger, D. A. Schkade, N. Schwarz, and A. A. Stone, "A survey method for characterizing daily life experience: The day reconstruction method," Science, vol. 306, no. 5702, pp. 1776-1780, 2004.

[15] C. N. Scollon, C.-K. Prieto, and E. Diener, "Experience sampling: Promises and pitfalls, strength and weaknesses," in Assessing well-being, Springer, 2009, pp. 157-180.

[16] I. Li, A. K. Dey, and J. Forlizzi, "Using context to reveal factors that affect physical activity," ACM Transactions on Computer-Human Interaction (TOCHI), vol. 19, no. 1, p. 7, 2012.

[17] Y.-H. Kim, J. H. Jeon, E. K. Choe, B. Lee, K. Kim, and J. Seo, "Timeaware: Leveraging framing effects to enhance personal productivity," in Proceedings of the SIGCHI conference on human factors in computing systems, 2016.

[18] J. Kopp, "Self-monitoring: A literature review of research and practice," in Social Work Research and Abstracts, Oxford University Press, vol. 24, 1988, pp. 8 20 .
[19] W. J. Korotitsch and R. O. Nelson-Gray, "An overview of self-monitoring research in assessment and treatment.," Psychological Assessment, vol. 11, no. 4, p. 415, 1999.

[20] I. Li, A. Dey, and J. Forlizzi, "A stage-based model of personal informatics systems," in Proceedings of the SIGCHI Conference on Human Factors in Computing Systems, ACM, 2010, pp. 557-566.

[21] S. Consolvo, D. W. McDonald, T. Toscos, M. Y. Chen, J. Froehlich, B. Harrison, P. Klasnja, A. LaMarca, L. LeGrand, R. Libby, et al., "Activity sensing in the wild: A field trial of ubifit garden," in Proceedings of the SIGCHI Conference on Human Factors in Computing Systems, ACM, 2008, pp. 1797-1806.

[22] M. L. Dontje, M. de Groot, R. R. Lengton, C. P. van der Schans, and W. P. Krijnen, "Measuring steps with the fitbit activity tracker: An inter-device reliability study," Journal of medical engineering \& technology, vol. 39, no. 5, pp. 286-290, 2015.

[23] J. J. Lin, L. Mamykina, S. Lindtner, G. Delajoux, and H. B. Strub, "Fish'n'steps: Encouraging physical activity with an interactive computer game," in International Conference on Ubiquitous Computing, Springer, 2006, pp. 261-278.

[24] E. K. Choe, B. Lee, M. Kay, W. Pratt, and J. A. Kientz, "Sleeptight: Low-burden, self-monitoring technology for capturing and reflecting on sleep behaviors," in Proceedings of the 2015 ACM International Joint Conference on Pervasive and Ubiquitous Computing, ACM, 2015, pp. 121-132.

[25] G. Lee, C. Tsai, W. G. Griswold, F. Raab, and K. Patrick, "Pmeb: A mobile phone application for monitoring caloric balance," in CHI'06 Extended Abstracts on Human Factors in Computing Systems, ACM, 2006, pp. 1013-1018.

[26] L. Mamykina, E. Mynatt, P. Davidson, and D. Greenblatt, "Mahi: Investigation of social scaffolding for reflective thinking in diabetes management," in Proceedings of the SIGCHI Conference on Human Factors in Computing Systems, ACM, 2008, pp. 477-486.

[27] (2017). Moves. Accessed: 9/3/2017.

[28] (2017). Fitbit. Accessed: 9/3/2017.

[29] R. Fleck and G. Fitzpatrick, "Reflecting on reflection: Framing a design landscape," in Proceedings of the 22nd Conference of the Computer-Human Interaction Special Interest Group of Australia on ComputerHuman Interaction, ACM, 2010, pp. 216-223.

[30] V. Braun and V. Clarke, "Using thematic analysis in psychology," Qualitative research in psychology, vol. 3, no. 2, pp. 77-101, 2006. 